\title{
Unusually large Mongolian blue spots in GM1 gangliosidosis: Is there an association?
}

\author{
Manouri P Senanayake $^{1}$, P V Dissanayake ${ }^{2}$, S. Rupasinghe ${ }^{2}$, J K K Seneviratne ${ }^{3}$
}

Sri Lanka Journal of Child Health, 2010; 39: 117-118

(Key words: Mongolian blue spots, GM1 gangliosidosis)

GM1 gangliosidosis is a rare storage disorder in which accumulation of sphingolipids occurs in brain and viscera due to a deficiency of a lysosomal enzyme. Multiple and unusually large Mongolian blue spots have been postulated to be an associated feature of lysosomal storage disorders ${ }^{1,2}$. Our patient, whose clinical course and physical signs were in keeping with GM1 gangliosidosis, had extensive Mongolian blue spots and this adds to the evidence supporting such an association.

\section{Case Report}

A seven month old girl, born by normal delivery to consanguineous parents, presented with developmental regression. Coarse facial features including low set ears, broad nasal bridge, a long philtrum and frontal bossing were present but there were no corneal opacities or macular cherry red spots. She had hepatosplenomegaly, hypotonia with brisk tendon reflexes, poor response to auditory stimuli and global developmental delay. A blueblack, maculopapular rash with clear margins resembling angiokeratoma had appeared symmetrically in the "bathing trunk area" at age four months (Figures 1, $2 \& 3$ ). Face, trunk and upper limbs were spared. In addition, large hyperpigmented, well demarcated macules resembling Mongolian blue spots were scatterd all over the body since birth.

Psychomotor regression coincided with the appearance of angiokeratoma. Urine was weakly positive for dermatan sulphate. Radiography showed anterior beaking of the lower part of vertebrae and oar shaped ribs. Beta galactosidase assay was not available but clinical differentiation from mucopolysaccharidosis (type 1) was possible, based on the absence of corneal opacities and the presence of angiokeratoma.

${ }^{1}$ Professor of Paediatrics, Department of Paediatrics, Faculty of Medicine, Colombo, ${ }^{2}$ Paediatric Registrar Lady Ridgeway Hospital, Colombo, ${ }^{3}$ Consultant Dermatologist, Lady Ridgeway Hospital, Colombo.

(Received on 14 July 2009. Accepted 20 August 2009)

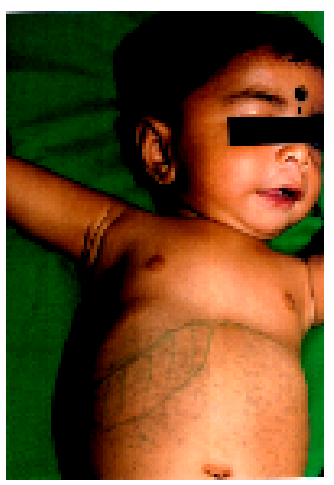

Figure 1: Coarse facial features, hepatomegaly and pigmented macules

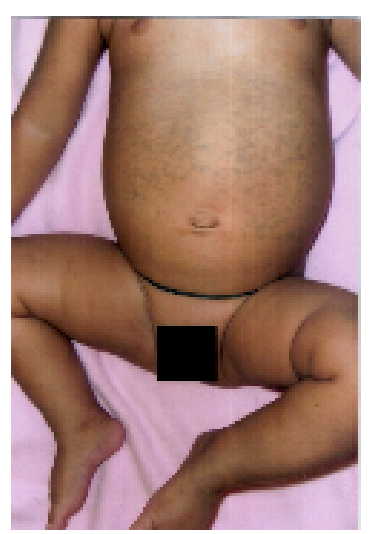

Figure 2: Typical angiokeratoma in bathing trunk area

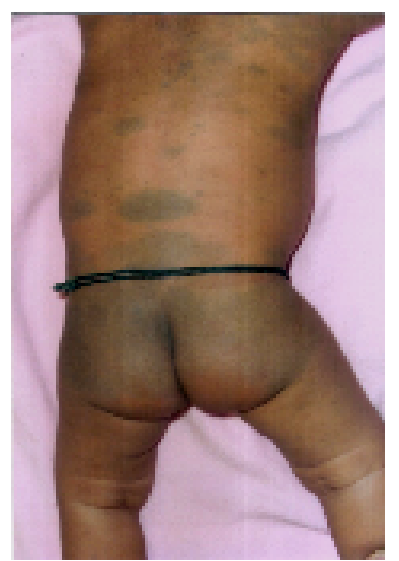

Figure 3: Extensive persistent Mongolian blue spots at age seven months 


\section{Discussion}

The presence of angiokeratoma is well recognised in several lysosomal disorders, most commonly in Fabry Disease and GM1 gangliosidosis. Female sex, age of onset of symptoms and presence of organomegaly excluded Fabry disease. Presence of angiokeratoma (Figure 2) helped in clinical differentiation of GM1 ganglioisidosis from mucopolysaccharidosis type 1 .

With the non availablity of enzyme assay, diagnosis of lysomal stoarge disorders heavily depends on clinical features. Therefore the observation of large, extensive and persistent Mongolian blue spots as an early sign warrants attention. Previous reports of this observation were disregarded as coincidental ${ }^{3,4,5,6}$ but recent reviews have postulated an association ${ }^{1,2}$. Our report adds support to this association.

In our patient large Mongolian blue spots present from birth persisted at seven months without evidence of fading. Such skin markings, otherwise considered benign, precede recognised clinical features of GM1 gangliosidosis. Establishing an association between unusally large and extensive Mongolian blue spots and lysosomal storage disorders, would enable early diagnosis of this inherited disorder for which treatment options are being developed and early initiation recommended for improved outcome ${ }^{2}$.

\section{References}

1. Hanson M, Lupski J R. Hicks J, Metry D. Association of dermal melanocytosis with lysosomal storage disease. Archives of Dermatology 2003: 139 (7): 916-20.

2. Ashrafi M, Shabanian R, Mohammadi M Kavusi S. Extensive Mongolian spots: A clinical sign which merits special attention. Pediatric Neurology, 2009; 34 (2): 143-5.

3. Selsor LC, Lesher JL. Hyperpigmented macules and patches in a child with GM1 gangliosidosis. Journal of American Academy of Dermatology 1989; 20 (5): 878-82.

4. Beratis NG, Varvariqou FA, Beratis S, Sklower SL. Angiokeratoma corporis diffusum. Clinical Genetics 1989; 36 (1): 59-64.

5. Weissbluth M, Esetrly NB, Caro WA. Report of an infant with GM1Gangliosidosis type 1 and extensive and unusual Mongolian blue spots. British Journal of Dermatology 1981; 104 (2): 195200.

6. Tegay, David. "GM1 Gangliosidosis”. Available from; www.emedicine.com/ped/topic2891.htm Accessed August 2004 